\title{
An Integrated Gateway for Various PHDs in U-Healthcare Environments
}

\author{
KeeHyun Park and JuGeon Pak \\ Computer Engineering Department, Keimyung University, Sindang-dong, Dalseo-gu, Daegu 704-701, Republic of Korea
}

Correspondence should be addressed to JuGeon Pak, corea@kmu.ac.kr

Received 16 May 2012; Accepted 10 June 2012

Academic Editor: Sabah Mohammed

Copyright () 2012 K. Park and J. Pak. This is an open access article distributed under the Creative Commons Attribution License, which permits unrestricted use, distribution, and reproduction in any medium, provided the original work is properly cited.

\begin{abstract}
We propose an integrated gateway for various personal health devices (PHDs). This gateway receives measurements from various PHDs and conveys them to a remote monitoring server (MS). It provides two kinds of transmission modes: immediate transmission and integrated transmission. The former mode operates if a measurement exceeds a predetermined threshold or in the case of an emergency. In the latter mode, the gateway retains the measurements instead of forwarding them. When the reporting time comes, the gateway extracts all the stored measurements, integrates them into one message, and transmits the integrated message to the MS. Through this mechanism, the transmission overhead can be reduced. On the basis of the proposed gateway, we construct a u-healthcare system comprising an activity monitor, a medication dispenser, and a pulse oximeter. The evaluation results show that the size of separate messages from various PHDs is reduced through the integration process, and the process does not require much time; the integration time is negligible.
\end{abstract}

\section{Introduction}

In recent years, the rapid emergence of population aging and chronic diseases on a global scale has contributed to social issues by increasing health insurance costs and lowering the quality of life. Consequently, many researchers are focusing on ubiquitous healthcare (u-healthcare).

Owing to this trend, personal health devices (PHDs) have emerged as key components of u-healthcare systems $[1,2]$. A PHD is a device that measures patient health data. Activity monitors, medication dispensers, pulse oximeters, and blood pressures are representative examples of PHDs.

In typical u-healthcare service architectures, PHDs measure and acquire patient health data and transmit them to a compute engine (CE), such as a smartphone or personal computer. A CE collects health data from PHDs and transmits them to a remote monitoring server (MS). The MS displays the received health data to medical staff, and it provides the patients with the analysis via a web-based UI.

In this way, communication protocols between PHDs and CEs are very important for collecting health data from various PHDs in a unified manner [3]. As a consequence, the
ISO/IEEE 11073 standard [4] was proposed to define how personal health data should be exchanged between a PHD and a CE, and what format should be used for the data. The standard specifies basic communication procedures and data formats to support any type of PHD. It also defines device specialization protocols in consideration of the characteristics of each PHD.

In general, a logical point-to-point channel is established between a PHD and a CE, and the PHD communicates with single $\mathrm{CE}$ at any point in time. A CE may communicate with more than one agent simultaneously using separate pointto-point connections. Therefore, the $\mathrm{CE}$ has a vital effect on the efficiency of the u-healthcare system. For this reason, we developed a handheld CE and designed two kinds of transmission modes: immediate transmission and integrated transmission in the previous studies $[3,5]$.

In this paper, we present the implementation results of an integrated gateway placed in a $\mathrm{CE}$. The gateway receives measurements from PHDs and conveys them to an MS. On the basis of the proposed gateway, we construct a u-healthcare system comprising three PHDs: an activity monitor [6], a medication dispenser [7], and a pulse oximeter [8]. Some 


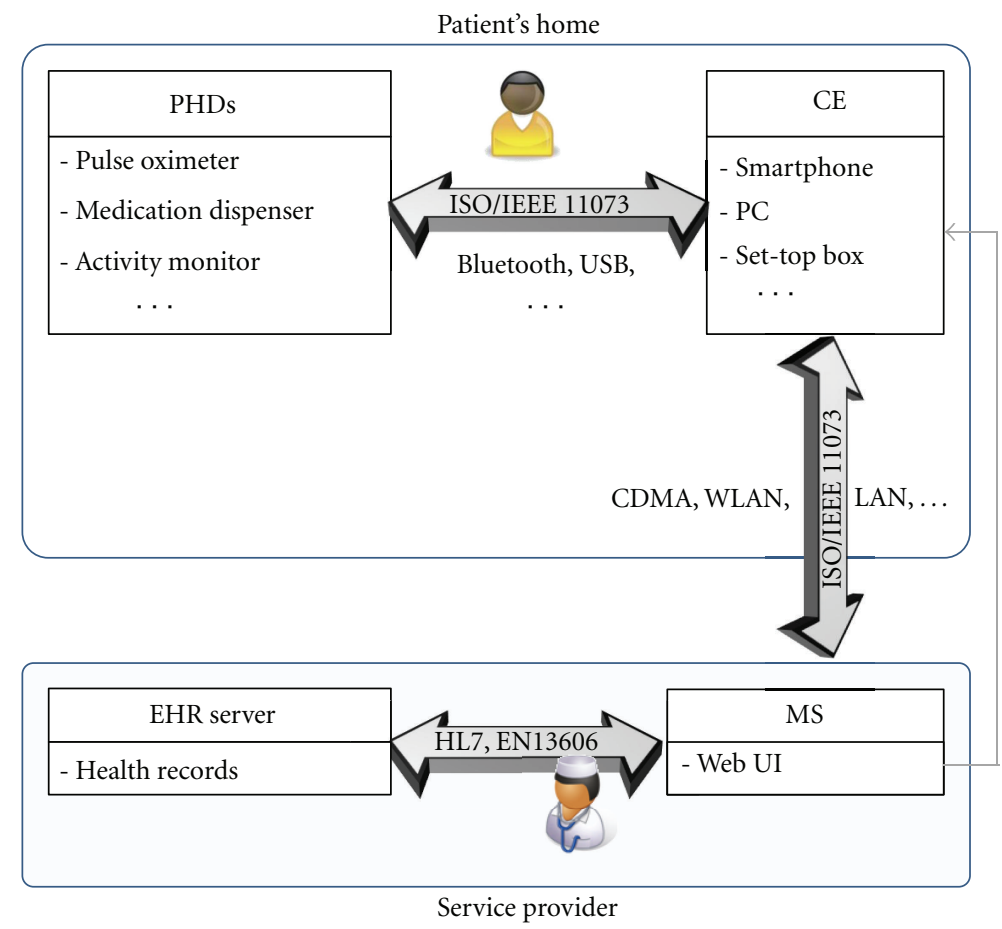

FiguRE 1: General architecture of u-healthcare system.

experiments show that the size of separate messages from these PHDs is reduced through the integration process, and the integration time does not increase significantly with an increase in the number of separate messages.

The remainder of this paper is organized as follows. Section 2 introduces the architecture of existing $\mathrm{u}$-healthcare systems and discusses their improvement on the basis of previous studies. Section 3 describes the proposed method and $\mathrm{u}$-healthcare system. Section 4 presents the application and experimental results of the proposed gateway, and Section 5 summarizes and concludes the paper.

\section{General Architecture of u-Healthcare System}

The architectures of $\mathrm{u}$-healthcare systems can be standardized or generalized by referring to previous studies on $\mathrm{u}$-healthcare systems. PHDs for measuring and acquiring patient health data have been proposed in the past [914]. Examples of PHDs are activity monitors, medication dispensers, pulse oximeters, and blood pressures. Some studies were conducted to construct a network for communication between components of a u-healthcare system. These studies proposed methods for applying body area networks (BANs), personal area networks (PANs), mobile networks (GSM, CDMA), wireless local area networks (WLANs), and Worldwide Interoperability for Microwave Access (WiMAX) [15-18] to u-healthcare systems. In addition, standard protocols for transparent integration and plug-and-play interoperability have been actively investigated [19-23]. The architectures of $\mathrm{u}$-healthcare systems can be generalized on the basis of these studies; the generalized architecture is shown in Figure 1.

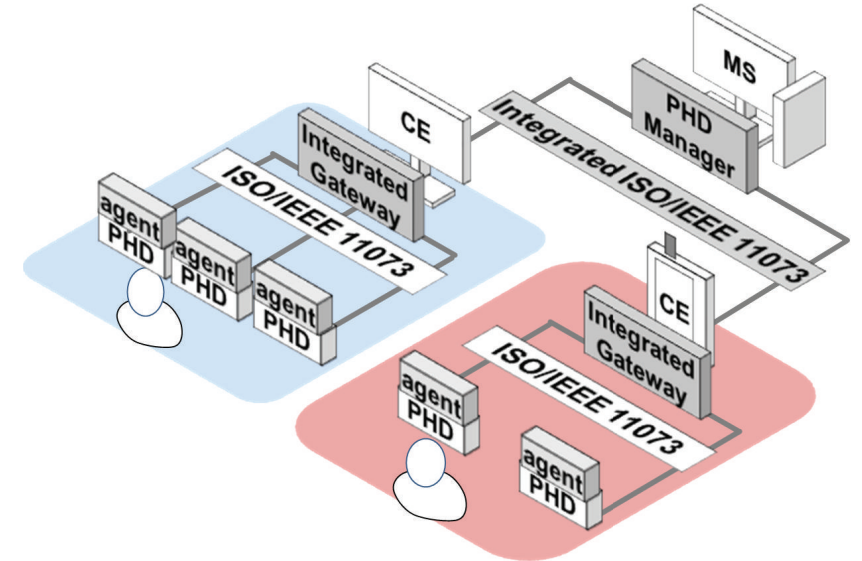

Figure 2: Architecture of the proposed u-healthcare system.

PHDs measure and acquire patient health data and transmit them to CEs via wired/wireless communication, for example, Bluetooth and USB. CEs collect health data from PHDs and transmit them to a remote MS via mobile communication (GSM or CDMA) or WLAN (WiFi). The MS displays the received health data to medical staff, and it provides the patients with the analysis via a web-based UI. An EHR server stores patient health data according to the EHR rules, but this is out of scope of this paper.

In this architecture, communication protocols among components are very important for collecting health data from various PHDs and CEs in a unified manner. As a consequence, ISO/IEEE 11073 [4] was developed to define how personal health data should be exchanged between 


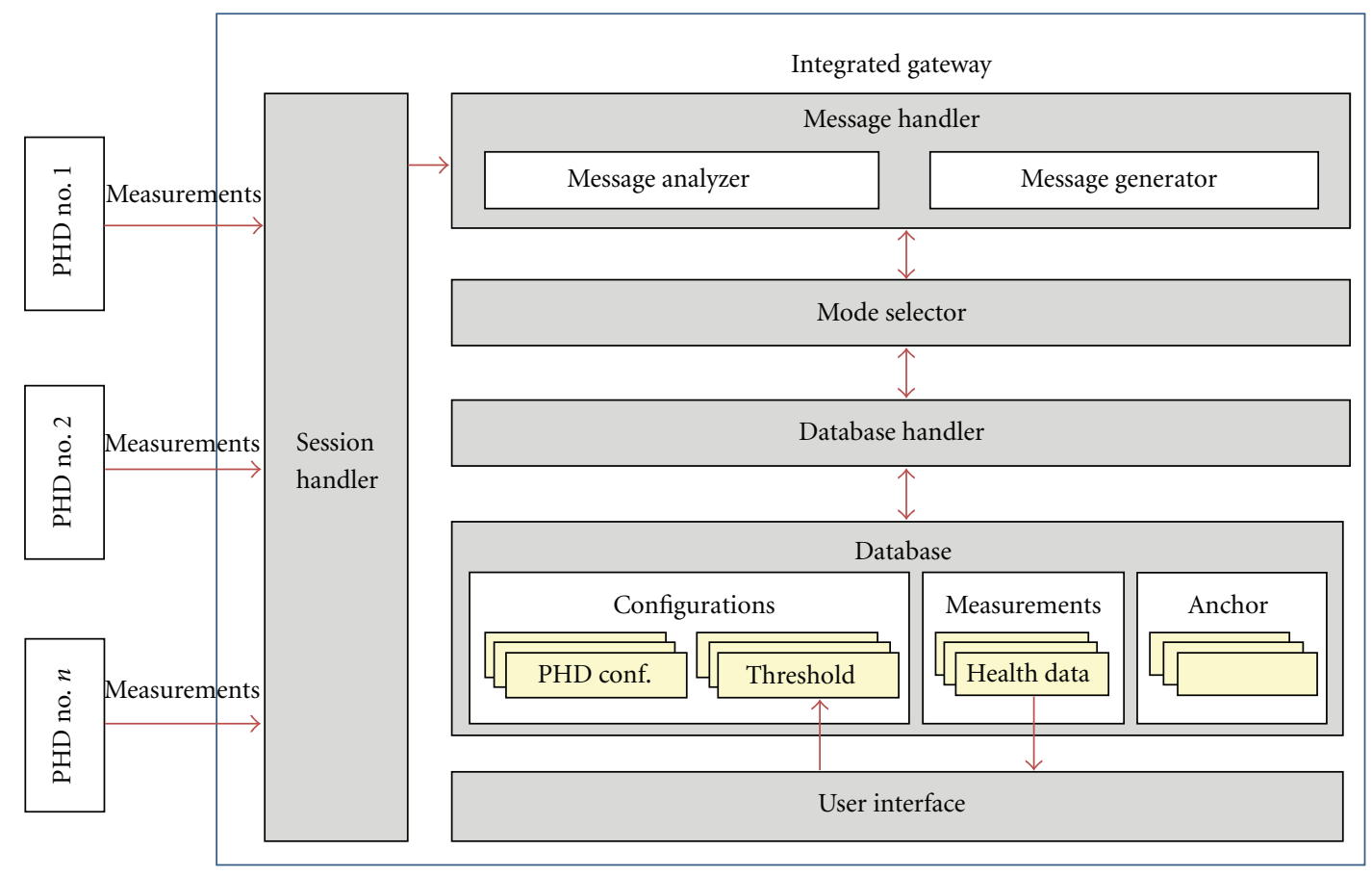

FIGURE 3: Architecture of an integrated gateway.

a PHD and a CE, and what format should be used for the data. The communication procedures are as follows.

(i) Association and setup phase: this phase associates communication sessions between a PHD and a CE. A PHD sends an association request message including association information such as system ID and configuration ID to CE. The CE analyzes the message, checks the configuration of the PHD, and sends an association response message to the PHD.

(ii) Operation phase: when a CE recognizes a PHD's configuration, this phase is initiated. During this phase, a PHD transmits measurements with a data report message.

(iii) Configuration phase: when the CE does not recognize the PHD's configuration, this phase is initiated. During this phase, the PHD sends its configuration to the CE.

(iv) Disassociation phase: this phase is for disassociating a communication session between a PHD and a CE.

In ISO/IEEE 11073, a logical point-to-point channel between a PHD and CE is established, and a PHD communicates with a single $\mathrm{CE}$ at any point in time. A CE may communicate with more than one agent simultaneously using separate point-to-point connections.

Measurements from each PHD have different characteristics. For example, $\mathrm{SpO}_{2}$, pulse rate, and number of steps are sensed continuously, whereas the status of some medications and fall detection are sensed intermittently. Measurement criticality also differs. A high level of $\mathrm{SpO}_{2}$, a high pulse rate, or misdosing may cause fatal consequences in patients. On the other hand, violation of an exercise program is a secondary risk. Therefore, a CE has to decide both which measurements should be transferred to an MS and when.

\section{Proposed u-Healthcare System}

3.1. Architecture of the Proposed u-Healthcare System. Figure 2 shows the architecture for our proposed $\mathrm{u}$ healthcare system. A PHD agent developed in a previous study [8] is assigned to each PHD, and this transfers the measurements to the CE. An integrated gateway is placed in the $\mathrm{CE}$ to decide both which measurements should be transferred to an MS and when. It also integrates the different kinds of 11073 messages received from PHDs into a single, integrated message, which is then transferred to an MS. PHD manager is assigned to an MS, and this analyzes the integrated message from the CE.

3.2. Integrated Gateway. To improve transmission efficiency, we implement an integrated gateway and place it in a CE. This gateway receives measurements from various $\mathrm{PHDs}$ and conveys them to an external MS. In this process, we apply two kinds of transmission modes: immediate transmission and integrated transmission [3]. In the former mode, the gateway immediately conveys the measurements it receives to an MS. This mode operates if a measurement exceeds a predetermined threshold or in the case of an emergency. Other than these cases, the gateway operates in the latter mode, whereby the measurements are stored instead of being forwarded. Then, when the reporting time comes, all the stored measurements are extracted and integrated into one message, and the integrated message is transmitted to the MS. Through this mechanism, the transmission overhead can be reduced. Figure 3 shows the architecture 


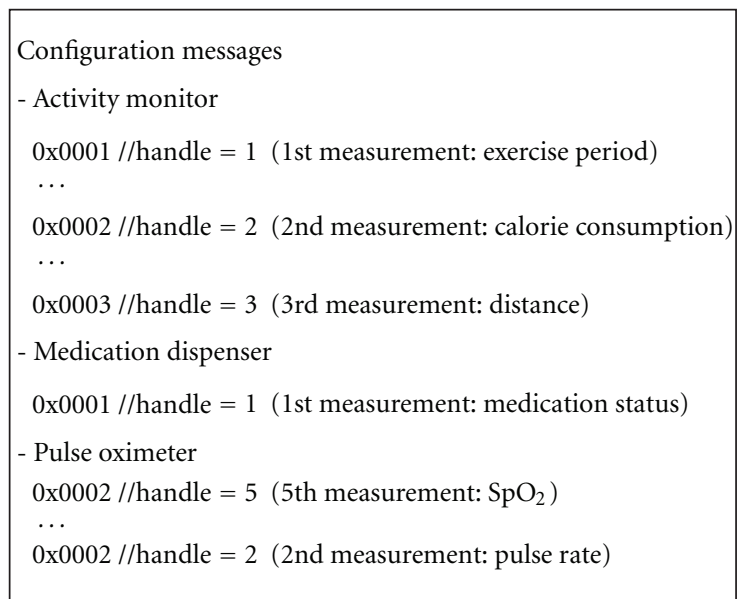

(a) Configuration messages transmitted from the PHDs to the $\mathrm{CE}$

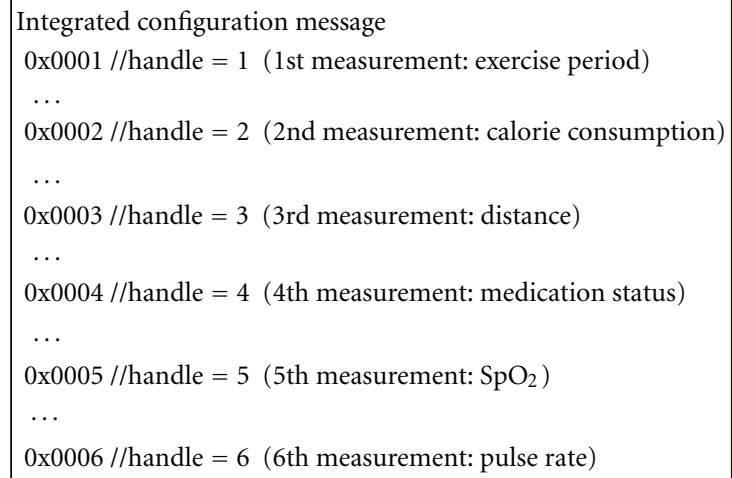

(b) Integrated configuration message transmitted from the CE to the MS

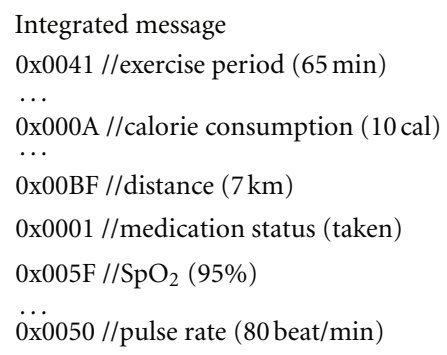

(c) Integrated message transmitted from the CE to the MS

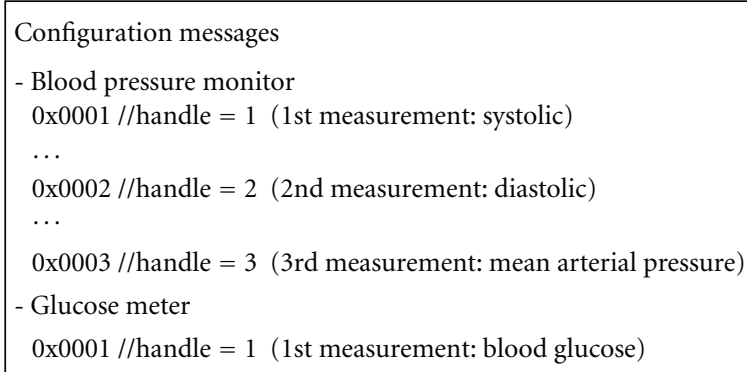

(d) Configuration messages transmitted from the added PHDs to the $\mathrm{CE}$

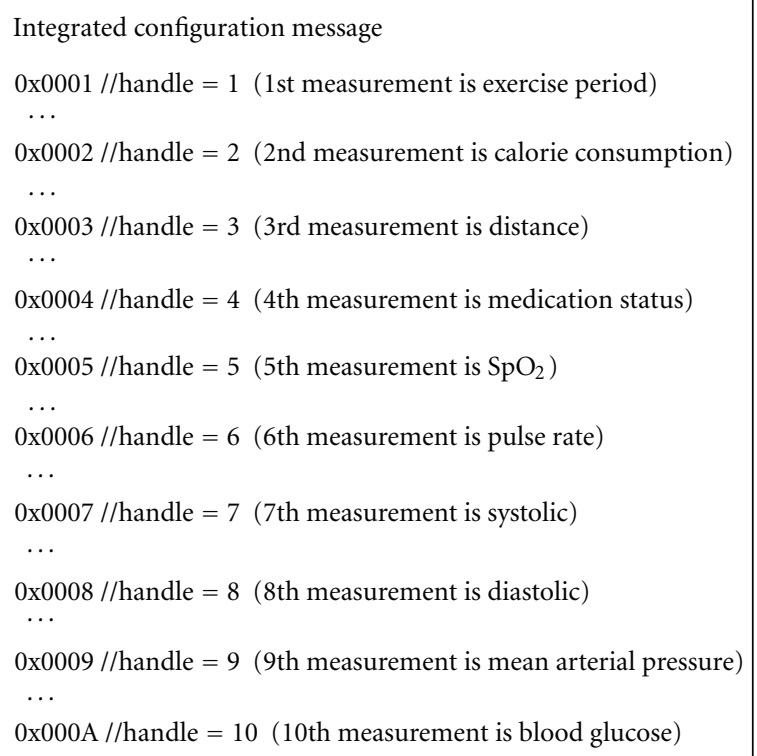

(e) Integrated configuration message transmitted from the CE to the MS

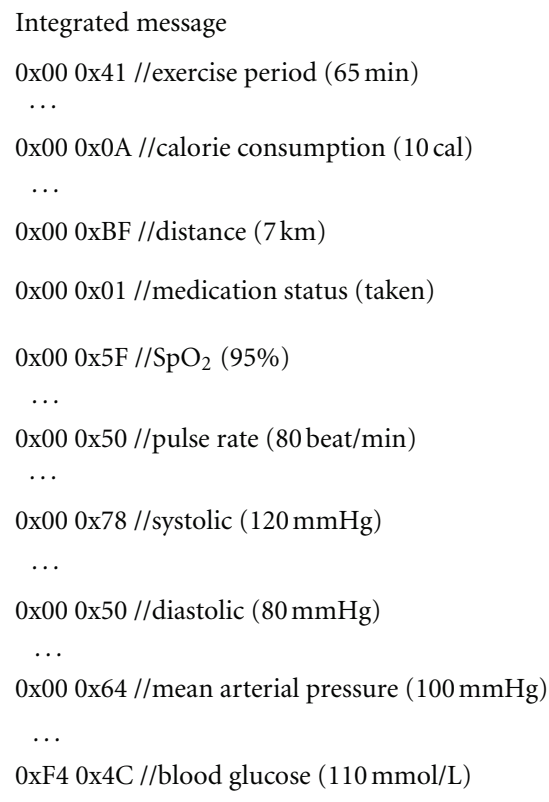

(f) Integrated message transmitted from the CE to the MS

FIGURE 4: Message integration scenario. 


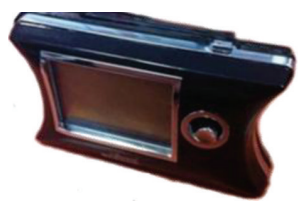

Activity monitor

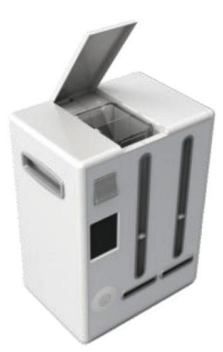

Medication dispenser

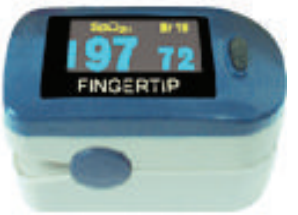

Pulse oximeter

Figure 5: Applied PHDs.
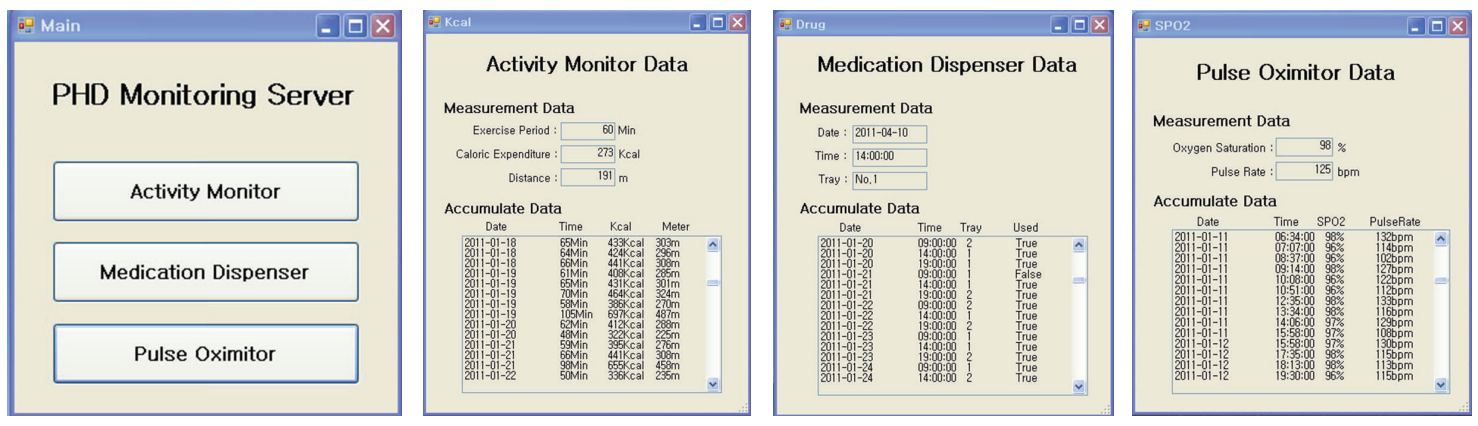

FIgURE 6: Graphical UI of MS.

of an integrated gateway. It consists of a session handler, message handler, mode selector, database handler, database, and user interface. The details of each handler are described in [3].

The optimized exchange protocol [24] provides a mechanism for static data (e.g., types of measurements and their units) to be transmitted only once, rather than on every data transmission, during the configuration phase. Once a configuration phase is negotiated between a PHD and CE, the PHD transfers only dynamic data (i.e., measured health data) to the CE. Therefore, the static data are excluded from the integration, and only the measurements are targeted by the integration.

In ISO/IEEE 11073, each PHD is represented as a medical device system (MDS). Measurements are represented as attributes of objects, and unique handles and attribute IDs are assigned to each object and attribute, respectively. However, uniqueness is only guaranteed within distinct PHDs, and there may be some duplication between different PHDs. For example, the object handle "1" can be assigned to the $\mathrm{SpO}_{2}$ object in a pulse oximeter while also assigned to the body weight object of a weighing scale. Therefore, object handles should be reassigned to guarantee global uniqueness, and the configuration phase between the CE and the MS enables this. For example, consider the scenario as shown in Figure 4. There are three PHDs (activity monitor, medication dispenser, and pulse oximeter) that belong to the same CE.

(1) The PHDs transmit configuration messages to the CE for negotiating (see Figure 4(a)).
(2) The CE re-assigns the value of the object handles, and transmits an integrated configuration message to the MS (see Figure 4(b)).

(3) After these processes, the CE can transmit the integrated message to the MS (see Figure 4(c)).

(4) If the two PHDs (blood pressure monitor and glucose meter) are added, these PHDs transmits their configuration messages to the CE (see Figure 4(d)).

(5) The CE reassigns the value of the object handles and transmits an integrated configuration message to the MS (see Figure 4(e)).

(6) After these processes, the CE can transmit the integrated message to the MS (see Figure 4(f)).

\section{Application and Experimental Results}

4.1. Application Results. On the basis of the proposed gateway, we construct a u-healthcare system comprising three PHDs: an activity monitor [6], a medication dispenser [7], and a pulse oximeter [8]. In addition, a CE and an MS implemented in the previous study [5] are extended and applied.

The activity monitor measures the user's physical exercise using a 3-axis accelerator, the medication dispenser delivers medication to chronic disease patients according to a predetermined schedule, and the pulse oximeter measures a user's $\mathrm{SpO}_{2}$ and pulse rate noninvasively. The 11073-10441 [25], 11073-10472 [26], and 11073-10404 [27] are applied to the agents in the activity monitor, medication dispenser, and 

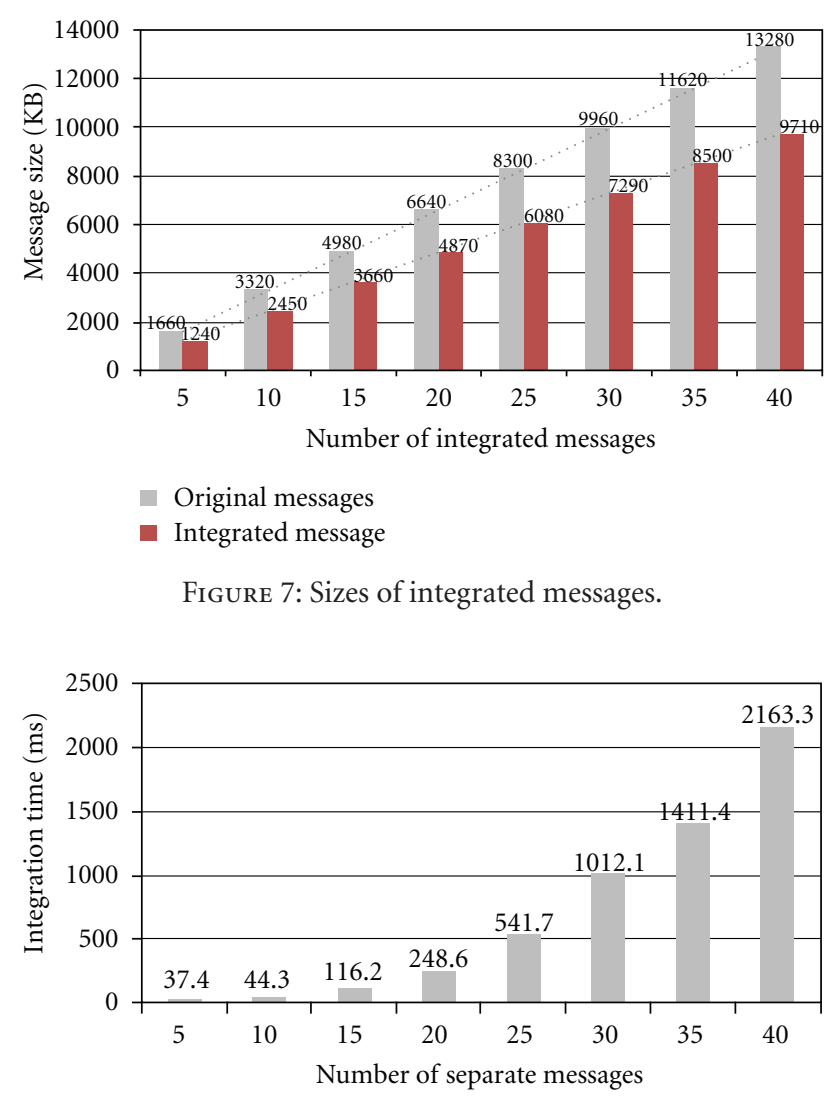

Figure 8: Integration times.

pulse oximeter, respectively. Figure 5 shows the PHDs used in our u-healthcare system.

The integrated gateway is implemented on the CE, and the MS is extended to monitor the applied PHDs. In this system, the CE and PHDs are connected via Bluetooth, and the CE and MS are connected via WiFi. Figure 6 shows the implemented and extended MS. Based on Figure 6, the MS receives an integration message from CE and displays measurements of each PHD properly.

4.2. Performance Evaluation. To verify the proposed gateway, the sizes of integrated messages and integration times were measured repeatedly as we increased the number of separate messages (measurements). The results of these evaluations are shown in Figures 7 and 8. Based on Figure 7, the gateway reduces the sizes of 5 separate messages and of 40 separate messages from 1,660 Kbytes to 1,240 Kbytes and from 13,280 Kbytes to 9,710 Kbytes, respectively.

Figure 8 shows the integration times of the proposed gateway. As shown in Figure 8, the gateway spends about $2.1 \mathrm{sec}$ for integrating 40 separate messages. According to the evaluations, we could verify that the proposed gateway in this paper is effective for $\mathrm{u}$-healthcare systems.

\section{Conclusion}

In this paper, we proposed an integrated gateway for various PHDs. This gateway receives measurements from various
PHDs and conveys them to a remote MS. We implemented two kinds of transmission modes: immediate transmission and integrated transmission. The former mode operates if a measurement exceeds a predetermined threshold or in the case of an emergency. In the latter mode, it retains the measurements instead of forwarding them. When the reporting time comes, the gateway extracts all the stored measurements, integrates them into one message, and transmits the integrated message to the MS. Through this mechanism, the transmission overhead can be reduced. On the basis of the proposed gateway, we constructed a uhealthcare system comprising an activity monitor, a medication dispenser, and a pulse oximeter. To verify the proposed gateway, the sizes of integrated messages and integration times were measured. According to the evaluations, we could verify that the proposed gateway in this paper is effective for u-healthcare systems.

In the future, we plan to apply the integrated gateway to other PHDs such as blood pressures and glucometers.

\section{Acknowledgment}

This research was supported by Basic Science Research Program through the National Research Foundation of Korea (NRF) funded by the Ministry of Education, Science and Technology (2011-0207).

\section{References}

[1] A. O'Brien and R. M. Ruairí, "Survey of assistive technology devices and applications for aging in place," in Proceedings of the 2nd International Conference on Advances in HumanOriented and Personalized Mechanisms, Technologies, and Services (CENTRIC'09), pp. 7-12, September 2009.

[2] A. Dittmar, F. Axisa, G. Delhomme, and C. Gehin, "New concepts and technologies in home care and ambulatory monitoring," Studies in Health Technology and Informatics, vol. 108, pp. 9-35, 2004.

[3] J. G. Pak and K. H. Park, "Design of an ISO/IEEE, 11073 gateway for u-healthcare services," in Proceedings of the International Conference on Information Science and Technology, pp. 152-154, 2012.

[4] IEEE Std. 1073 Standard for Medical Device Communication-Part 00000: Framework and Overview.

[5] K. H. Park and J. G. Pak, "Implementation of a handheld compute engine for personal health devices," International Journal of Smart Home, vol. 6, no. 2, pp. 59-64, 2012.

[6] J. Pak and K. Park, "A smart personal activity monitoring system based on wireless device management methods," Communications in Computer and Information Science, vol. 184, no. 1, pp. 335-342, 2011.

[7] J. G. Pak and K. H. Park, "UbiMMS: an ubiquitous medication monitoring system based on remote device management methods," The Journal of Healthcare Information Management, vol. 41, no. 1, pp. 26-30, 2012.

[8] J. G. Pak and K. H. Park, "Pulse oximeter monitor for uhealth service," in Proceeding of the International Conference on Computer and Applications, p. 61, 2012.

[9] J. B. J. Bussmann, W. L. J. Martens, J. H. M. Tulen, F. C. Schasfoort, H. J. G. van den Berg-Emons, and H. J. Stam, "Measuring daily behavior using ambulatory accelerometry: 
The activity monitor," Behavior Research Methods, Instruments, and Computers, vol. 33, no. 3, pp. 349-356, 2001.

[10] J. J. Segura-Juárez, D. Cuesta-Frau, L. Samblas-Pena, and M. Aboy, "A microcontroller-based portable electrocardiograph recorder," IEEE Transactions on Biomedical Engineering, vol. 51, no. 9, pp. 1686-1690, 2004.

[11] V. F. S. Fook, A. A. P. Wai, M. Jayachandran, B. Jit, P. H. Lee, and P. Y. L. Kiat, "Smart mote-based wireless medication management system for persons with dementia," International Journal of Assistive Robotics and Mechatronics, vol. 9, no. 1, pp. 27-35, 2008.

[12] P. H. Tsai, T. Y. Chen, C. R. Yu, C. S. Shih, and J. W. S. Liu, "Smart medication dispenser: design, architecture and implementation," IEEE Systems Journal, vol. 5, no. 1, pp. 99111, 2011.

[13] T. Pedersen, P. B. Dyrlund, and A. M. Moller, "Pulse oximetry for perioperative monitoring," Cochrane Database of Systematic Reviews, vol. 3, Article ID CD002013, 2003.

[14] N. Watthanawisuth, T. Lomas, A. Wisitsoraat, and A. Tuantranont, "Wireless wearable pulse oximeter for health monitoring using ZigBee wireless sensor network," in Proceedings of the 7th Annual International Conference on Electrical Engineering/Electronics, Computer, Telecommunications and Information Technology (ECTI-CON '10), pp. 575-579, May 2010.

[15] P. Harvey, B. Woodward, S. Datta, and D. Mulvaney, "Data acquisition in a wireless diabetic and cardiac monitoring system," in Proceedings of the Engineering in Medicine and Biology Society, pp. 3154-3157, 2011.

[16] D. Niyato, E. Hossain, and S. Camorlinga, "Remote patient monitoring service using heterogeneous wireless access networks: architecture and optimization," IEEE Journal on Selected Areas in Communications, vol. 27, no. 4, pp. 412-423, 2009.

[17] I. Martiìnez, P. del Valle, P. Munoz et al., "Interoperable and standard e-health solution over Bluetooth," in Proceedings of the Engineering in Medicine and Biology Society, pp. 21922195, 2010.

[18] D. Benhaddou, M. Balakrishnan, and X. Yuan, "Remote healthcare monitoring system architecture using sensor networks," in Proceedings of the IEEE Region 5 Conference, pp. 1-6, April 2008.

[19] D. P. Bogia, "Supporting personal health devices through standardization and collaboration," in Proceedings of the 13th IEEE International Conference on e-Health Networking Applications and Services, pp. 338-343, 2011.

[20] M. Galarraga, I. Martinez, L. Serrano et al., "Proposal of an ISO/IEEE11073 platform for healthcare telemonitoring: plugand-play solution with new use cases," in Proceedings of the Engineering in Medicine and Biology Society, pp. 10-13, 2007.

[21] I. Martinez, J. Fernandez, M. Galarraga et al., "Implementation of an end-to-end standard-based patient monitoring solution," IET Communications, vol. 2, no. 2, pp. 6426-6429, 2008.

[22] M. Galarraga, L. Serrano, I. Martinez, P. de Toledo, and M. Reynolds, “Telemonitoring systems interoperability challenge: an updated review of the applicability of ISO/IEEE, 11073 standards for interoperability in telemonitoring," in Proceedings of the Engineering in Medicine and Biology Society, pp. 6162-6166.

[23] M. Clarke, D. Bogia, K. Hassing, L. Steubesand, T. Chan, and D. Ayyagari, "Developing a standard for personal health devices based on 11073," in Proceedings of the Engineering in Medicine and Biology Society, pp. 6175-6177, 2007.
[24] IEEE Std. 11073-20601-2008 Health Informatics-Personal Health Device Communication-Application ProfileOptimized Exchange Protocol.

[25] IEEE Std. 11073-10441-2008 Health Informatics-Personal Health Device Communication-Device specializationCardiovascular fitness and activity monitor.

[26] IEEE Std. 11073-10472-2010 Health Informatics-Personal Health Device Communication-Device specializationMedication Monitor.

[27] IEEE Std. 11073-10404-2008 Health Informatics-Personal Health Device Communication-Device specializationPulse oximeter. 

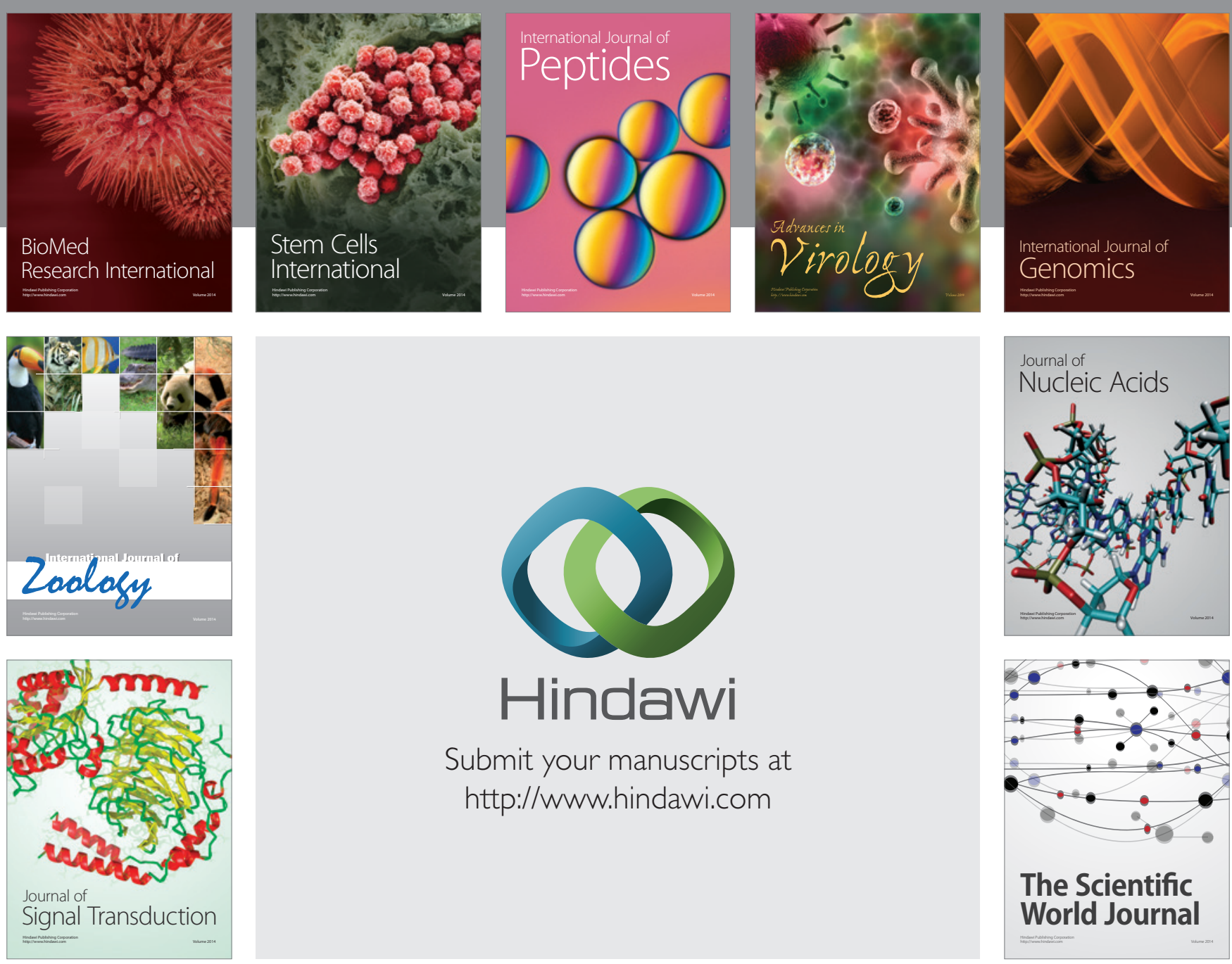

Submit your manuscripts at

http://www.hindawi.com
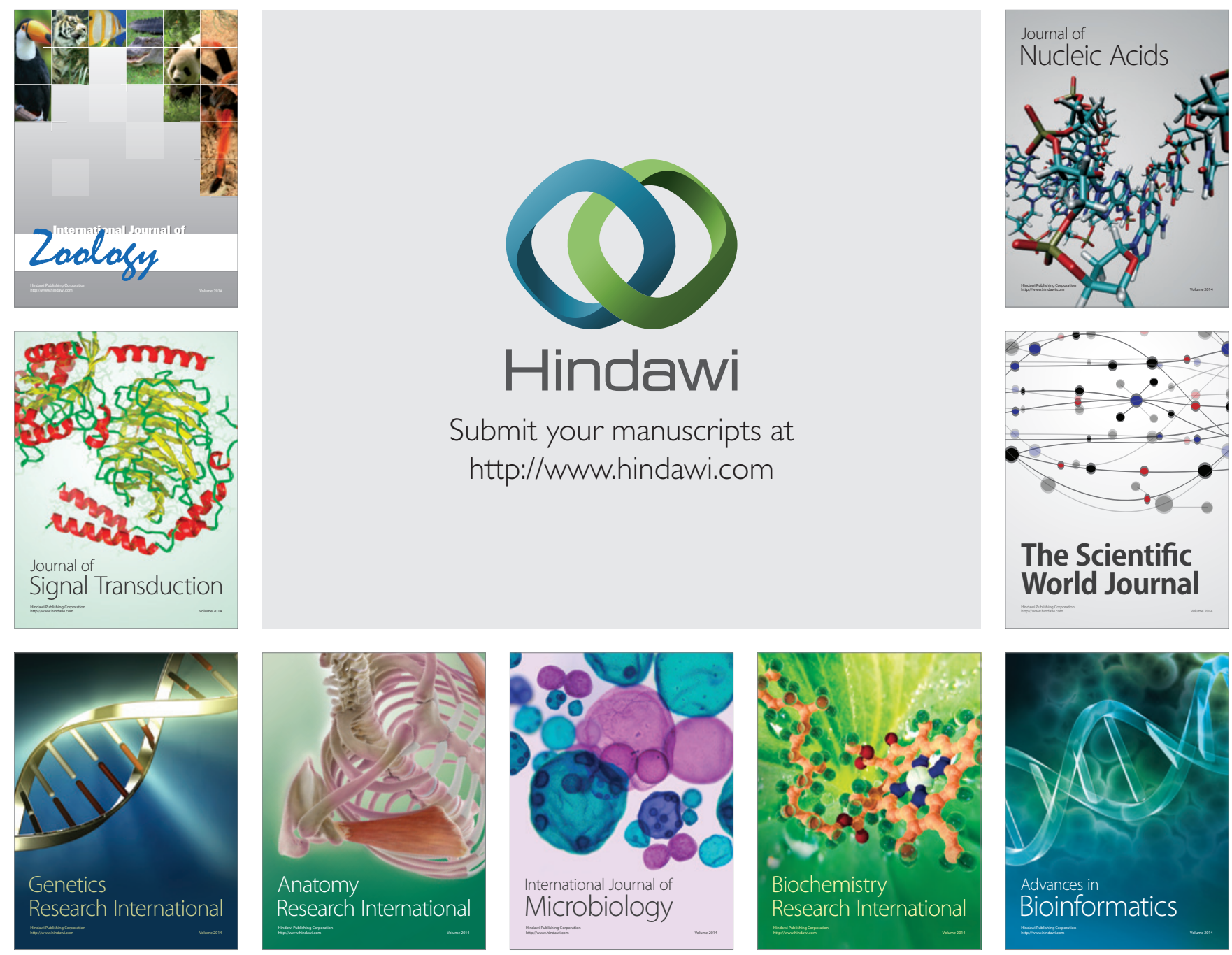

The Scientific World Journal
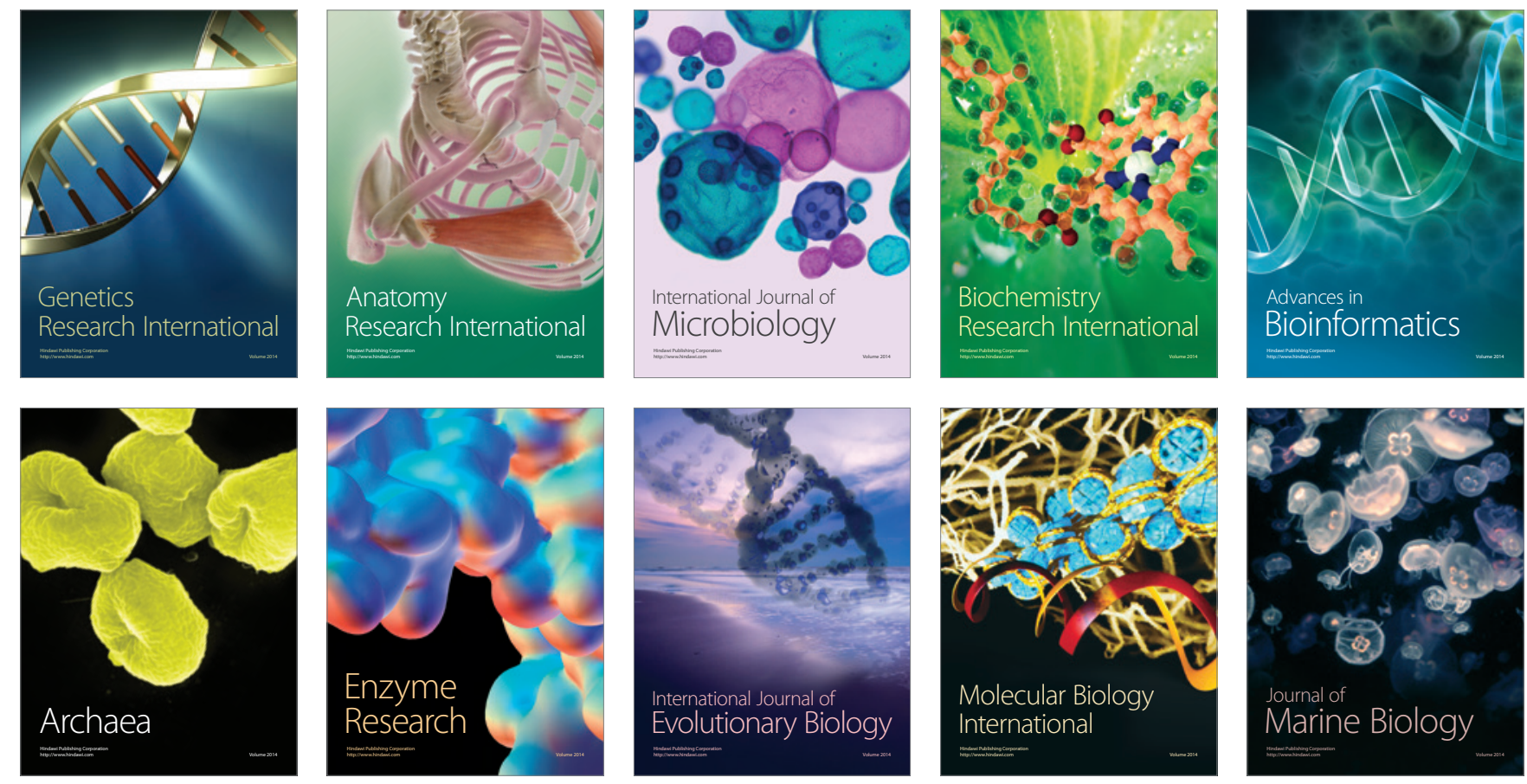